\title{
The divisor problem for arithmetic progressions with small modulus
}

\author{
by
}

\author{
Charles E. Chace (Williamstown, Mass.)
}

Notation. The symbol " $=$ " indicates that the statement is a definition. The notation $f^{l}(x)$ means $(f(x))^{l}$. The symbols "«" or " $\langle-\rangle$ " mean the same thing as "«" or "O(-)" except that the implied "constant" may contain $\varepsilon$-powers of the variables involved; e.g. $\log x \lll 1$. If $E(a)$ is an expression involving $a$, then the expression $E\left(a^{-}\right)$means $\lim _{\varepsilon \rightarrow 0} E(a-\varepsilon)$.

1. Introduction. Let $d_{l}(n)$ denote the $l$ th divisor function, that is, the number of ordered $l$-tuples of positive integers whose product is equal to $n$. Then we define

$$
D_{l}(x ; a, q):=\sum_{\substack{n \leq x \\ n \equiv a \bmod q}} d_{l}(n) .
$$

This can be written in the form

$$
D_{l}(x ; a, q)=M_{l}(x ; a, q)+\Delta_{l}(x ; a, q),
$$

and the divisor problem for arithmetic progressions involves estimating the remainder term. The elementary bound $\Delta_{l}(x ; a, q) \lll_{l} x^{1-1 / l}+q^{l-1}$ is given in [C]. Nowak [No] estimates $\Delta_{l}(x ; a, q)$ for fixed $q$, while Petečuk [P] allows $q$ to be an arbitrary power of a fixed odd prime; in both papers the emphasis is on obtaining the smallest possible bound for the error term, just as in the usual divisor problem. Most of the work on this problem, however, concentrates on finding upper bounds for $q$ for which the remainder is smaller than the main term; see for example Smith [S], Matsumoto [Ma], Heath-Brown [H3] and Friedlander and Iwaniec, [FI1] and [FI2]. Lavrik [L1] gives an estimate for $\Delta_{l}(x ; a, q)$ with a parameter so that one can adjust the contributions from $x$ and $q$. In many of these papers the bound given is worse than the elementary bound when $q$ is small compared with $x$.

The main term $M_{l}(x ; a, q)$ can be expressed in a number of ways. It can be written in the form $x P(\log x)$ where $P(z)$ is a polynomial of degree 
$l-1$. Using Perron's formula, it can be expressed as a residue of a certain meromorphic function, as in [H3], [FI1], [FI2] and [S], and there is yet another interpretation given in [L1]. In the case $l=2$, Kopetzky $[\mathrm{K}]$ gives an expression in terms of Euler constants for arithmetic progressions and Motohashi [Mo] gives one involving Ramanujan sums. In the case $q=1$, Lavrik [L2] writes $M_{l}(x ; a, q)$ in terms of the Laurent coefficients of $\zeta(s)$.

The purpose of this paper is to give an analogue of the result of [L2], and to estimate the remainder term for $q$ relatively small. Our results are as follows:

THEOREM 1. The main term has the form

$$
M_{l}(x ; a, q)=\sum_{n=0}^{l-1} c_{n+1}(a, q) L_{n}(x)
$$

where the coefficients $c_{n}(a, q)$ are given in (2.13), satisfy $c_{n}(a, q) \lll_{l} q^{-1}$, and

$$
L_{n}(x):=x \sum_{j=0}^{n}(-1)^{n-j} \frac{\log ^{j} x}{j !} ;
$$

note that $L_{n}^{\prime}(x)=\left(\log ^{n} x\right) / n !$.

TheOrem 2. Let $l \geq 4$ be an integer, and suppose $(a, q)=1$. Then

$$
\Delta_{l}(x ; a, q) \lll_{l} x^{(l-1) /(l+2)}+x^{1 / 2} q^{(l-4) / 4} .
$$

Note that the second term here is worse than the trivial bound $x q^{-1}$ for $q \geq x^{2 / l}$.

Proposition 3. Suppose

$$
\Delta_{l}(x ; a, q) \lll_{l} x^{\eta} q^{\theta}
$$

for $q \leq x^{\kappa}$, provided $(a, q)=1$. Then if $(a, q)=k$ we have

$$
\Delta_{l}(x ; a, q) \lll_{l} x^{\eta} q^{\theta} k^{-(\eta+\theta)}
$$

for $q \leq x^{\kappa} k^{1-\kappa l}$. Furthermore, if there is no restriction on $q$ in the case $(a, q)=1$, then there will be no restriction when $(a, q)>1$.

If we apply this to the bound in Theorem 2, we obtain the bound

$$
\Delta_{l}(x ; a, q) \lll_{l}\left(\frac{x}{k}\right)^{(l-1) /(l+2)}+\left(\frac{x}{k}\right)^{1 / 2}\left(\frac{q}{k}\right)^{(l-4) / 4} ;
$$

the first term dominates the second when $q \leq x^{2 /(l+2)} k^{l /(l+2)}$.

The proof of Theorem 1 is done in Sections 2, 3 and 4. In Section 2 we derive the expression for $M_{l}(x ; a, q)$ given in the theorem; the $c_{n}(a, q)$ are expressed in terms of the Laurent coefficients of a "Hurwitz-like" zeta 
function

$$
\zeta(s ; a, q):=\sum_{\substack{n \geq 1 \\ n \equiv a \bmod q}} \frac{1}{n^{s}} .
$$

In Section 3 we write the Laurent coefficients of $\zeta(s ; a, q)$ in a form which we can estimate, and in Section 4 we derive the stated bounds for $c_{n}(a, q)$. In Section 5 we prove Theorem 2 by imitating the argument of $[\mathrm{T}], \S 12.3$, obtaining a bound for $\Delta_{l}(x ; a, q)$ on condition that $a$ and $q$ are relatively prime; in Section 6 we extend a technique given by Heath-Brown in the case $l=3$ ([H3], §8) for removing this condition to prove Proposition 3.

This paper consists mostly of results from my doctoral dissertation, and I wish to acknowledge the inestimable help of my advisor, P. X. Gallagher, and express my deepest gratitude. I must also acknowledge helpful suggestions made by William Duke, David Witte, and an anonymous referee, all of which contributed to this paper in its final form.

\section{PROOF OF THEOREM 1}

2. A residue computation. Smith gives the expression

$$
M_{l}(x ; a, q)=\operatorname{Res}\left\{Z_{l}(s ; a, q) \frac{x^{s}}{s} ; s=1\right\}
$$

([S], Theorem 3) where

$$
Z_{l}(s ; a, q):=\sum_{\substack{n \geq 1 \\ n \equiv a \bmod q}} \frac{d_{l}(n)}{n^{s}} .
$$

We write this in terms of

$$
\zeta(s ; a, q):=\sum_{\substack{n \geq 1 \\ n \equiv a \bmod q}} \frac{1}{n^{s}},
$$

namely

$$
Z_{l}(s ; a, q)=\sum_{a_{1} \ldots a_{l} \equiv a \bmod q} \zeta\left(s ; a_{1}, q\right) \ldots \zeta\left(s ; a_{l}, q\right),
$$

and then express $M_{l}(x ; a, q)$ in terms of the Laurent coefficients of $\zeta(s ; a, q)$. By an argument completely analogous to the one used for the Riemann zeta function, we obtain a Laurent expansion of the form

$$
\zeta(s ; a, q)=\frac{1}{q} \frac{1}{s-1}+\sum_{m=0}^{\infty} \gamma_{m}(a, q)(s-1)^{m} ;
$$

see the proof of Proposition 3.1. 
First we write $x^{s} / s$ as a power series around $s=1$ :

$$
\frac{x^{s}}{s}=\sum_{m=0}^{\infty} L_{m}(x)(s-1)^{m}
$$

where

$$
L_{m}(x):=x \sum_{j=0}^{m}(-1)^{m-j} \frac{\log ^{j} x}{j !}
$$

note that $L_{m}^{\prime}(x)=\left(\log ^{m} x\right) / m !$.

We now compute $M_{l}(x ; a, q)$; the following notation will make the result easier to state. We define the "convolution $\bmod q$ " of $f$ and $g$ by

$$
f *_{q} g(a):=\sum_{\substack{a_{1} a_{2} \equiv a \bmod q \\ 1 \leq a_{1}, a_{2} \leq q}} f\left(a_{1}\right) g\left(a_{2}\right) .
$$

The operation $*_{q}$ is clearly commutative and associative, and so we have

$$
f^{*_{q} j}(a):=\underbrace{f *_{q} \ldots *_{q} f}_{j \text { times }}(a)=\sum_{a_{1} \ldots a_{j} \equiv a \bmod q} f\left(a_{1}\right) \ldots f\left(a_{j}\right) .
$$

From (2.4) and (2.5) we have

$$
\begin{array}{r}
Z_{l}(s ; a, q)=\sum_{a_{1} \ldots a_{l} \equiv a \bmod q}\left(\frac{1}{q} \frac{1}{s-1}+\sum_{m=0}^{\infty} \gamma_{m}\left(a_{1}, q\right)(s-1)^{m}\right) \ldots \\
\ldots\left(\frac{1}{q} \frac{1}{s-1}+\sum_{m=0}^{\infty} \gamma_{m}\left(a_{l}, q\right)(s-1)^{m}\right)
\end{array}
$$

and multiplying out gives, in the notation of (2.8),

$$
\begin{aligned}
& Z_{l}(s ; a, q)=\frac{1}{(s-1)^{l}} \frac{1^{*_{q} l}(a)}{q^{l}} \\
& +\sum_{n=1}^{l-1} \frac{1}{(s-1)^{n}} \sum_{i=n}^{l-1} \sum_{\boldsymbol{j} \in \boldsymbol{D}}\left(\begin{array}{c}
l \\
i, j
\end{array}\right) \frac{1^{*_{q} i} *_{q} \gamma_{0} *_{q} j_{0} *_{q} \ldots *_{q} \gamma_{i-n} *_{q} j_{i-n}(a, q)}{q^{i}} \\
& + \text { holomorphic }
\end{aligned}
$$

where $\boldsymbol{j}=\left\{j_{0}, \ldots, j_{i-n}\right\}$ is a vector whose components are non-negative integers,

$$
\boldsymbol{D}:=\left\{\boldsymbol{j}: i+\sum_{k=0}^{i-n} j_{k}=l, \sum_{k=1}^{i-n} k j_{k}=i-n\right\}
$$


and for $i+\sum_{k=0}^{m} j_{k}=l$,

$$
\left(\begin{array}{c}
l \\
i, \boldsymbol{j}
\end{array}\right):=\frac{l !}{i ! j_{0} ! \ldots j_{m} !} .
$$

Multiplying (2.6) and (2.10) we see that

$$
M_{l}(x ; a, q)=\sum_{n=0}^{l-1} c_{n+1}(a, q) L_{n}(x),
$$

where

$$
\begin{aligned}
c_{l}(a, q) & :=\frac{1^{*_{q} l}(a)}{q^{l}} \\
c_{n}(a, q) & :=\sum_{i=n}^{l-1} \sum_{\boldsymbol{j} \in \boldsymbol{D}}\left(\begin{array}{c}
l \\
i, \boldsymbol{j}
\end{array}\right) \frac{1^{*_{q} i} *_{q} \gamma_{0}{ }^{*} j_{0} *_{q} \ldots *_{q} \gamma_{i-n}{ }^{*_{q} j_{i-n}}(a, q)}{q^{i}}
\end{aligned}
$$

for $1 \leq n \leq l-1$.

3. An expression for $\gamma_{m}(a, q)$. In this section we derive an expression for $\gamma_{m}(a, q)$ which will allow us to estimate the coefficients $c_{n}(a, q)$. Let $a$ and $q$ be integers with $q \geq 1$ and $1 \leq a \leq q$. Then we define

$$
C_{m}(a, q):=\lim _{x \rightarrow \infty}\left(\sum_{\substack{n \leq x \\ n \equiv a \bmod q}} \frac{(\log n)^{m}}{n}-\frac{1}{q} \frac{(\log x)^{m+1}}{m+1}\right) .
$$

In Lemma 3.1 we show that the limit exists, indeed how rapidly it converges. The main result of this section is the following

Proposition 3.1. Let $\gamma_{m}(a, q)$ be as in (2.5). Then

$$
\gamma_{m}(a, q)=\frac{(-1)^{m}}{m !} C_{m}(a, q) .
$$

We require two lemmas, the first of which is a preliminary fact about approximating sums by integrals, cf. $[\mathrm{T}], 2.1 .2$. Let $\psi(x):=x-[x]-1 / 2$.

Lemma 3.0. Let $f$ be continuous on the interval $[u, v]$. Then

$$
\begin{aligned}
\sum_{\substack{u<n \leq v \\
n \equiv a \bmod q}} f(n)= & \frac{1}{q} \int_{u}^{v} f(y) d y \\
& +\int_{u}^{v} \psi\left(\frac{y-a}{q}\right) d f(y)+\psi\left(\frac{u-a}{q}\right) f(u)-\psi\left(\frac{v-a}{q}\right) f(v) .
\end{aligned}
$$


Pr o of. Denote the sum by $S$. In $S$, let $n=m q+a$. Then

$$
\begin{aligned}
S & =\sum_{(u-a) / q<m \leq(v-a) / q} f(m q+a) \\
& =\int_{(u-a) / q}^{(v-a) / q} f(q x+a) d x-\int_{(u-a) / q}^{(v-a) / q} f(q x+a) d \psi(x) .
\end{aligned}
$$

We then substitute $y=q x+a$ and integrate the second term by parts.

LEMma 3.1. We have

(a)

$$
C_{0}(a, q)=\int_{1^{-}}^{\infty}\left\{\psi\left(\frac{y-a}{q}\right)-\psi\left(\frac{1^{-}-a}{q}\right)\right\} d\left(\frac{1}{y}\right)
$$

and for $m \geq 1$,

$$
C_{m}(a, q)=\int_{1^{-}}^{\infty} \psi\left(\frac{y-a}{q}\right) d\left(\frac{(\log y)^{m}}{y}\right) .
$$

Further, for all $m \geq 0$,

(c) $\sum_{\substack{n \leq x \\ n \equiv a \bmod q}} \frac{\log ^{m} n}{n}=\frac{1}{q} \frac{\log ^{m+1} x}{m+1}+C_{m}(a, q)+O_{m}\left(\frac{\log ^{m} x}{x}\right)$.

Proof. First, let $m=0$ and apply Lemma 3.0 with $f(y)=1 / y$ to get

$$
\begin{aligned}
\sum_{\substack{n \leq x \\
n \equiv a \bmod q}} \frac{1}{n}= & \frac{1}{q} \int_{1^{-}}^{x} \frac{1}{y} d y \\
& +\int_{1^{-}}^{x} \psi\left(\frac{y-a}{q}\right) d\left(\frac{1}{y}\right)+\psi\left(\frac{1^{-}-a}{q}\right)-\psi\left(\frac{x-a}{q}\right) \frac{1}{x} .
\end{aligned}
$$

Note that

$$
\int_{x}^{\infty} \psi\left(\frac{y-a}{q}\right) d\left(\frac{1}{y}\right) \ll \frac{1}{x}
$$

and that

$$
\int_{1}^{\infty} d\left(\frac{1}{y}\right)=-1
$$

so we can write

$$
\psi\left(\frac{1^{-}-a}{q}\right)=-\int_{1}^{\infty} \psi\left(\frac{1^{-}-a}{q}\right) d\left(\frac{1}{y}\right)
$$


and hence

$$
\sum_{\substack{n \leq x \\ n \equiv a \bmod q}} \frac{1}{n}=\frac{1}{q} \log x+\int_{1^{-}}^{\infty}\left\{\psi\left(\frac{y-a}{q}\right)-\psi\left(\frac{1^{-}-a}{q}\right)\right\} d\left(\frac{1}{y}\right)+O\left(\frac{1}{x}\right) .
$$

From the definition (3.1) of $C_{m}(a, q)$ we have

$$
C_{0}(a, q)=\int_{1^{-}}^{\infty}\left\{\psi\left(\frac{y-a}{q}\right)-\psi\left(\frac{1^{-}-a}{q}\right)\right\} d\left(\frac{1}{y}\right)
$$

and the lemma is proved.

If $m \geq 1$ then Lemma 3.0 gives

$$
\begin{aligned}
\sum_{\substack{n \leq x \\
n \equiv a \bmod q}} \frac{\log ^{m} n}{n}= & \frac{1}{q} \int_{1^{-}}^{x} \frac{\log ^{m} y}{y} d y \\
& +\int_{1^{-}}^{x} \psi\left(\frac{y-a}{q}\right) d\left(\frac{\log ^{m} y}{y}\right)-\psi\left(\frac{x-a}{q}\right) \frac{\log ^{m} x}{x} .
\end{aligned}
$$

The above argument shows that

$$
C_{m}(a, q)=\int_{1^{-}}^{\infty} \psi\left(\frac{y-a}{q}\right) d\left(\frac{\log ^{m} y}{y}\right)
$$

and also proves the lemma.

Proof of Proposition 3.1. We imitate the argument of Titchmarsh; see $[T], 2.1 .16$. First we note that

$$
\gamma_{m}(a, q)=\frac{1}{m !} f^{(m)}(1)
$$

where

$$
f(s):=\zeta(s ; a, q)-\frac{1}{q} \frac{1}{s-1} .
$$

For $\operatorname{Re} s>1$ we have

$$
\zeta^{(m)}(s ; a, q)=(-1)^{m} \sum_{\substack{n \geq 1 \\ n \equiv a \bmod q}} \frac{\log ^{m} n}{n^{s}} .
$$

Applying Lemma 3.0 gives

$$
\begin{array}{r}
\zeta^{(m)}(s ; a, q)=(-1)^{m}\left\{\frac{1}{q} \int_{1}^{\infty} \frac{\log ^{m} y}{y^{s}} d y+\int_{1^{-}}^{\infty} \psi\left(\frac{y-a}{q}\right) d\left(\frac{\log ^{m} y}{y^{s}}\right)\right. \\
\left.+\psi\left(\frac{1^{-}-a}{q}\right) \log ^{m}(1)\right\}
\end{array}
$$


for $m \geq 0$. By integration by parts and induction we have

$$
\int_{1}^{\infty} \frac{\log ^{m} y}{y^{s}} d y=\frac{m !}{(s-1)^{m+1}}
$$

and so

$$
\gamma_{m}(a, q)=\frac{(-1)^{m}}{m !}\left\{\int_{1^{-}}^{\infty} \psi\left(\frac{y-a}{q}\right) d\left(\frac{\log ^{m} y}{y}\right)+\psi\left(\frac{1^{-}-a}{q}\right) \log ^{m}(1)\right\}
$$

and we are finished by Lemma 3.1 .

4. An estimate for $c_{n}(a, q)$. In this section we give an estimate for $\gamma_{m}(a, q)$ and for expressions of the form $1^{*_{q}}{ }^{i} *_{q} G_{j}(a, q)$ where $G_{j}$ is a convolution $\bmod q$ of $j$ factors, each of which is $\gamma_{m}(a, q)$ for some $m$; these occur in the expression (2.13) for $c_{n}(a, q)$.

LEMMA 4.1. We have

$$
C_{m}(a, q)=\frac{\log ^{m} a}{a}+O_{m}\left(\frac{\log ^{m+1} q}{q}\right) ;
$$

in particular,

$$
C_{m}(a, q) \lll a^{-1} .
$$

P r o of. We start from Lemma 3.1(a) or (b) and write

$$
\int_{1^{-}}^{\infty}=\int_{1^{-}}^{a}+\int_{a}^{q}+\int_{q}^{\infty} \text {. }
$$

For the last of these we note that

$$
\int_{q}^{\infty}\left|d\left(\frac{\log ^{m} y}{y}\right)\right| \ll_{m} \frac{\log ^{m} q}{q} .
$$

We now use the fact that

$$
\psi(x)= \begin{cases}x+1 / 2 & \text { if }-1 \leq x<0, \\ x-1 / 2 & \text { if } 0 \leq x<1\end{cases}
$$

Suppose $m \geq 1$. Then from Lemma 3.1(b) and (4.1) and (4.2) we have

$$
\begin{aligned}
C_{m}(a, q) & =\int_{1}^{a}\left(\frac{y-a}{q}+\frac{1}{2}\right) d\left(\frac{\log ^{m} y}{y}\right)+\int_{a}^{q}\left(\frac{y-a}{q}-\frac{1}{2}\right) d\left(\frac{\log ^{m} y}{y}\right) \\
& +O_{m}\left(\frac{\log ^{m} q}{q}\right) \\
= & \frac{\log ^{m} a}{a}+\frac{1}{q} \int_{1}^{q} y d\left(\frac{\log ^{m} y}{y}\right)+O_{m}\left(\frac{\log ^{m} q}{q}\right)
\end{aligned}
$$


on expanding and collecting similar terms, and absorbing what we can in the $O$-term. One then verifies by direct calculation that

$$
\int_{1}^{q} y d\left(\frac{\log ^{m} y}{y}\right) \ll_{m} \log ^{m+1} q .
$$

A similar argument, using Lemma 3.1(a) instead of (b), proves the lemma when $m=0$.

Remark. From this and Proposition 3.1 we see that

$$
\gamma_{m}(a, q) \lll_{m} a^{-1} .
$$

Lemma 4.2. Let 1(a) denote the function whose value is 1 for all a. Then

$$
1^{*_{q} 2}(a)=\sum_{a_{1} a_{2} \equiv a \bmod q} 1 \lll q
$$

and

(b)

$$
1 *_{q} C_{m}(a, q)=\sum_{a_{1} a_{2} \equiv a \bmod q} C_{m}\left(a_{1}, q\right) \lll_{m} 1 .
$$

Proof. We use the fact that the equation $a_{1} a_{2} \equiv a \bmod q$ has solutions in $a_{2}$ if and only if $\left(a_{1}, q\right) \mid a$. Let $a_{0}$ be the least solution satisfying $1 \leq a_{0} \leq$ $q$; then there are exactly $\left(a_{1}, q\right)$ solutions, namely

$$
a_{0}+j \frac{q}{\left(a_{1}, q\right)}
$$

for $0 \leq j \leq\left(a_{1}, q\right)-1$. Hence we can write

$$
\begin{aligned}
\sum_{a_{1} a_{2} \equiv a \bmod q} 1 & =\sum_{\substack{a_{1}=1 \\
\left(a_{1}, q\right) \mid a}}^{q}\left(a_{1}, q\right)=\sum_{d \mid a, q} d \sum_{c=1}^{q / d} 1=\sum_{d \mid a, q} d \phi\left(\frac{q}{d}\right) \\
& \leq q d((a, q)) \lll q
\end{aligned}
$$

and

$$
\begin{aligned}
\sum_{a_{1} a_{2} \equiv a \bmod q} C_{m}\left(a_{1}, q\right) & =\sum_{d \mid a, q} d \sum_{c=1}^{q / d} C_{m}(c d, q) \\
\oiiint_{m} & \sum_{\text {Lemma } 4.1)} d \sum_{d \mid a, q}^{q / d}(c d)^{-1} \ll d((a, q)) \log q \lll 1 .
\end{aligned}
$$

Remarks. (I) In (b), we can replace $C_{m}(a, q)$ by $\left|C_{m}(a, q)\right|$. 
(II) In the proof we use a special case of the fact that

$$
1 *_{q} f(a)=\sum_{a_{1} a_{2} \equiv a \bmod q} f\left(a_{1}\right)=\sum_{d \mid a, q} d \sum_{c=1}^{q / d} f(c d) ;
$$

note from this that $1 *_{q} f(a)$ depends only on $(a, q)$.

Proposition 4.1. Let $i$ and $j$ be integers with $i \geq 1$ and $j \geq 0$, and let $G_{j}(a, q)$ be a convolution $\bmod q$ of $j$ factors, each of which is $\gamma_{m}(-, q)$ for some $m$. Then

$$
1^{*_{q} i} *_{q} G_{j}(a, q) \lll_{i, j} q^{i-1} .
$$

Proof. Assume $j=0$. If $i=1$, the statement is trivial. If we assume

$$
1^{*_{q}(i-1)}(a) \lll_{i} q^{i-2}
$$

then

$$
1^{*_{q} i}(a)=\sum_{a_{1} a_{2} \equiv a \bmod q} 1^{*_{q}(i-1)}(a) \lll_{i} q^{i-2} \sum_{a_{1} a_{2} \equiv a \bmod q} 1 \lll_{i} q^{i-1}
$$

by Lemma $4.2(\mathrm{a})$ and the lemma is proved.

We now estimate $G_{j}(a, q)$ using Lemma 4.1:

$$
\begin{aligned}
G_{j}(a, q) & =\sum_{a_{1} \ldots a_{j} \equiv a \bmod q} \gamma_{m_{1}}\left(a_{1}, q\right) \ldots \gamma_{m_{j}}\left(a_{j}, q\right) \\
& \lll \sum_{a_{1} \ldots a_{j} \equiv a \bmod q} \frac{1}{a_{1} \ldots a_{j}} \lll \sum_{\substack{m \leq q^{j} \\
m \equiv a \bmod q}} \frac{d_{j}(m)}{m} .
\end{aligned}
$$

Using the fact that $d_{j}(m) \lll_{j} 1$, Lemma $3.1(\mathrm{c})$ with $x=q^{j}$ and Lemma 4.1 give

and so

$$
G_{j}(a, q) \lll_{j} \frac{1}{a},
$$

$$
1 *_{q} G_{j}(a, q) \lll_{j} \sum_{a_{1} a_{2} \equiv a \bmod q} \frac{1}{a_{1}} .
$$

From Remark (II) at the end of Lemma 4.2 we have

$$
1 *_{q} G_{j}(a, q) \lll_{j} \sum_{d \mid a, q} d \sum_{c=1}^{q / d} \frac{1}{c d} \lll 1 .
$$

To finish we proceed by induction as above.

Applying Proposition 4.1 to the expressions for $c_{n}(a, q)$ at the end of Section 2 gives the bound stated in Theorem 1. 
5. Proof of Theorem 2. In the author's thesis $[\mathrm{C}]$, Theorem 1, the elementary bound

$$
\Delta_{l}(x ; a, q) \lll_{l} x^{1-1 / l}+q^{l-1}
$$

was derived by mimicking the argument in $[\mathrm{T}], \S 12.1$. In the case $(a, q)=1$, Matsumoto's result [Ma] implies that

$$
\Delta_{l}(x ; a, q) \lll_{l} x^{(l-1) /(l+1)}
$$

if $q \leq x^{2 /(l+1)}$. By imitating the argument in [T], $\S 12.3$, we prove Theorem 2 , which we restate:

Theorem 2. Let $l \geq 4$ be an integer, and suppose $(a, q)=1$. Then

$$
\Delta_{l}(x ; a, q) \lll_{l} x^{(l-1) /(l+2)}+x^{1 / 2} q^{(l-4) / 4} .
$$

This improves (5.2) for $l \geq 4$ wherever that bound is non-trivial. The proof requires the following lemma, due to P. X. Gallagher.

Local Convexity Lemma. For $f$ analytic in the rectangle $\alpha \leq \sigma \leq \beta$, $|t| \leq \tau$ we have

$$
f(\sigma) \ll_{\alpha, \beta} A^{(\beta-\sigma) /(\beta-\alpha)} B^{(\sigma-\alpha) /(\beta-\alpha)},
$$

where

$$
A=\max _{|t| \leq \tau}|f(\alpha+i t)|, \quad B=\max _{|t| \leq \tau}|f(\beta+i t)|,
$$

provided $e^{\tau^{2}} \geq C / \min \{A, B\}$; here $C=\max _{\alpha \leq \sigma \leq \beta}|f(\sigma \pm i t)|$.

Proof. Let $g_{\sigma}(w)=f(w) y^{w-\sigma} e^{(w-\sigma)^{2}}$, with $y>0$ to be chosen later. Then $f(\sigma)=g_{\sigma}(\sigma)$ and for $w=u+i v$,

$$
g_{\sigma}(w) \ll \begin{cases}y^{\alpha-\sigma} A & \text { for } u=\alpha,|v| \leq \tau, \\ y^{\beta-\sigma} B & \text { for } u=\beta,|v| \leq \tau, \\ \max \left\{y^{\alpha-\sigma}, y^{\beta-\sigma}\right\} e^{-\tau^{2} C} & \text { for } \alpha \leq \sigma \leq \beta,|v|=\tau .\end{cases}
$$

By the maximum principle,

$$
f(\sigma) \ll \max \left\{y^{\alpha-\sigma} A, y^{\beta-\sigma} B, \max \left\{y^{\alpha-\sigma}, y^{\beta-\sigma}\right\} e^{-\tau^{2}} C\right\} .
$$

Taking $y^{\beta-\alpha}=A / B$ balances the first two items here, and under the final proviso in the statement of the lemma the third term is smaller.

Proof of Theorem 2. Following the argument of [H2], §8, we have

$$
\begin{aligned}
& \Delta_{l}(x ; a, q) \\
& =\frac{1}{2 \pi i} \int_{1 / 2-i T}^{1 / 2+i T} z(s) \frac{x^{s}}{s} d s+O\left(\int_{1 / 2 \pm i T}^{1+\varepsilon \pm i T} z(s) \frac{x^{s}}{s} d s\right)+\left\langle x(q T)^{-1}+1\right\rangle
\end{aligned}
$$


where

$$
z(s):=Z_{l}(s ; a, q)-\frac{d_{l}(a)}{a^{s}}-\frac{d_{l}(b)}{b^{s}},
$$

$b$ being the nearest integer to $x$ satisfying $b \equiv a \bmod q$.

Consider one of the "horizontal" integrals

$$
H:=\int_{1 / 2+i T}^{1+\varepsilon+i T} z(s) \frac{x^{s}}{s} d s
$$

the argument for the other will be the same. We will apply the local convexity lemma to $z(s)$ on the translated rectangle $1 / 2 \leq \sigma \leq 1+\varepsilon,|t-T| \leq \tau$. For $(a, q)=1$ we have

$$
Z_{l}(s ; a, q)=\frac{1}{\phi(q)} \sum_{\chi \bmod q} \bar{\chi}(a) L(s, \chi)^{l} .
$$

From Heath-Brown's hybrid bound [H1], Corollary 2, and Linnik's fourth power moment bound (see [Mn], p. 74) we have

$$
z\left(\frac{1}{2}+i t\right) \lll \mathcal{B}(t)^{l-4} t,
$$

where $\mathcal{B}(t)=(q t)^{1 / 6}+q^{1 / 4}$, and

$$
z(1+\varepsilon+i t) \lll q^{-1},
$$

following the analogous argument for $f(s)$ in [H2], p. 412. If $\tau \lll 1$ then $L(s, \chi) \lll(q T)^{2}$ on the top and bottom of the rectangle, and so $z(s) \lll$ $(q T)^{2 l}$ and the final proviso of the lemma is satisfied when $\tau \asymp_{l} \sqrt{\log q T}$. Hence we have

$$
z(\sigma+i T) \lll \mathcal{B}(T)^{2(l-4) l(1-\sigma)} T^{2(1-\sigma)} q^{1-2 \sigma}
$$

and so

$$
H \lll x(q T)^{-1}+x^{1 / 2} \mathcal{B}(T)^{l-4} .
$$

Now consider the "vertical" integral

$$
V:=\frac{1}{2 \pi i} \int_{1 / 2-i T}^{1 / 2+i T} Z_{l}(s ; a, q) \frac{x^{s}}{s} d s .
$$

The difference between $V$ and the first term on the right in (5.3) is

$$
\frac{1}{2 \pi i} \int_{1 / 2-i T}^{1 / 2+i T}\left(\frac{d_{l}(a)}{a^{s}}+\frac{d_{l}(b)}{b^{s}}\right) \frac{x^{s}}{s} d s \lll\left(\frac{x}{a}\right)^{1 / 2} .
$$

We use (5.4) and the hybrid bound again to write, for $l \geq 4$,

$$
V \lll \frac{x^{1 / 2}}{\phi(q)} \mathcal{B}(T)^{l-4} \sum_{\chi \bmod q} \int_{-T}^{T}\left|L\left(\frac{1}{2}+i t, \chi\right)\right|^{4} \frac{d t}{t+1} \lll x^{1 / 2} \mathcal{B}(T)^{l-4} ;
$$


for the second estimate here we integrate by parts and apply Montgomery's fourth-power-moment bound [Mn], Theorem 10.1.

Recalling the definition of $\mathcal{B}(T)$ above, note that for $T \geq q^{1 / 2}, \mathcal{B}(T) \ll$ $(q T)^{1 / 6}$. Under this restriction, combining (5.3) and the bounds on $H$ and $V$ we have

$$
\Delta_{l}(x ; a, q) \lll_{l} x(q T)^{-1}+x^{1 / 2}(q T)^{(l-4) / 6} .
$$

Choosing $T=x^{3 /(l+2)} q^{-1}$ gives

$$
\Delta_{l}(x ; a, q) \lll_{l} x^{(l-1) /(l+2)}
$$

and the restriction on $T$ is satisfied when $q \leq x^{2 /(l+2)}$. If instead we assume $T \leq q^{1 / 2}$ then

$$
\Delta_{l}(x ; a, q) \lll_{l} x(q T)^{-1}+x^{1 / 2} q^{(l-4) / 4}
$$

in which case we take $T=q^{1 / 2}$ and so

$$
\Delta_{l}(x ; a, q) \lll_{l} x q^{-3 / 2}+x^{1 / 2} q^{(l-4) / 4}
$$

and we finish by noting that the second term here dominates the first term and the bound in (5.5) precisely when $q \geq x^{2 /(l+2)}$.

6. Proof of Proposition 3. We use a technique given by Heath-Brown in the case $l=3$ (see [H3], §8) to remove the condition $(a, q)=1$. The result is the following

Proposition 3. Suppose

$$
\Delta_{l}(x ; a, q) \lll_{l} x^{\eta} q^{\theta}
$$

for $q \leq x^{\kappa}$, provided $(a, q)=1$. Then if $(a, q)=k$ we have

$$
\Delta_{l}(x ; a, q) \lll_{l} x^{\eta} q^{\theta} k^{-(\eta+\theta)}
$$

for $q \leq x^{\kappa} k^{1-\kappa l}$. Furthermore, if there is no restriction on $q$ in the case $(a, q)=1$, then there will be no restriction when $(a, q)>1$.

R e mark. Note that if $\kappa \leq 1 / l$ the restriction on $q$ for $k>1$ is no worse than the restriction when $k=1$.

The proof requires a lemma; let

$$
F_{l}(n, k):=\sum_{a_{1} \ldots a_{l} b=n}\left(\prod_{i=1}^{l} \mu\left(a_{i}\right)\right) d_{l}(b k) .
$$

We have the following

LEMmA 6.1. Let $k=(a, q)$, and let $a_{1}=a / k, q_{1}=q / k$ and $n \bar{n} \equiv$ $1 \bmod q_{1}$. Then

$$
D_{l}(x ; a, q)=\sum_{\left(n, q_{1}\right)=1} F_{l}(n, k) D_{l}\left(x / n k ; a_{1} \bar{n}, q_{1}\right) .
$$


Proof. We require the following facts about $F_{l}(n, k)$; if $\left(n_{1} k_{1}, n_{2} k_{2}\right)=$ 1 , then

and if $\alpha \geq l$, then

$$
\begin{aligned}
F_{l}\left(n_{1} n_{2}, k_{1} k_{2}\right) & =F_{l}\left(n_{1}, k_{1}\right) F_{l}\left(n_{2}, k_{2}\right), \\
F_{l}\left(p^{\alpha}, 1\right) & = \begin{cases}1 & \text { if } \alpha=0, \\
0 & \text { if } \alpha>0,\end{cases}
\end{aligned}
$$

$$
F_{l}\left(p^{\alpha}, p^{\beta}\right)=0 .
$$

The proof of the first of these is straightforward. Using the fact that

$$
d_{l}\left(p^{\alpha}\right)=\left(\begin{array}{c}
\alpha+l-1 \\
l-1
\end{array}\right),
$$

the proofs of the second and third facts reduce to the following identities:

$$
\sum_{j=0}^{\alpha}(-1)^{j}\left(\begin{array}{c}
\alpha-j+l-1 \\
l-1
\end{array}\right)\left(\begin{array}{l}
l \\
j
\end{array}\right)=0
$$

if $\alpha>0$, and

$$
\sum_{j=0}^{l}(-1)^{j}\left(\begin{array}{c}
\gamma-j+l-1 \\
l-1
\end{array}\right)\left(\begin{array}{l}
l \\
j
\end{array}\right)=0
$$

if $\gamma \geq l$.

Let $n=\prod p_{j}^{\nu_{j}}$ and $k=\prod p_{j}^{\kappa_{j}}$. Then $F_{l}(n, k)=0$ if $\nu_{j}>0$ and $\kappa_{j}=0$ or if $\nu_{j} \geq l$ and $\kappa_{j}>0$ for some $j$, i.e. unless for all $j$ we have $\nu_{j}=0$ or $\kappa_{j}>0$, and $\nu_{j}<l$ or $\kappa_{j}=0$; these conditions imply that $n \mid k^{l-1}$. By a straightforward imitation of Heath-Brown's argument for [H3], (8.4) and (8.5), we have for $(a, q)=1$,

$$
\sum_{(n, q)=1} F_{l}(n, k) D_{l}(x / n ; a \bar{n}, q)=\sum_{\substack{m \leq x \\ m \equiv a \bmod q}} \sum_{n \mid m} F_{l}(n, k) d_{l}(m / n)
$$

(note from the argument above that the sum on the left is finite) and

$$
\sum_{n \mid m} F_{l}(n, k) d_{l}(m / n)=d_{l}(m k),
$$

and so

$$
D_{l}(x k ; a k, q k)=\sum_{(n, q)=1} F_{l}(n, k) D_{l}(x / n ; a \bar{n}, q)
$$

and we finish by renaming variables.

Proof of Proposition 3. We now write $D_{l}=M_{l}+\Delta_{l}$ in the right-hand side of the statement of Lemma 6.1. Assuming that

$$
M_{l}(x ; a, q)=\sum_{\left(n, q_{1}\right)=1} F_{l}(n, k) M_{l}\left(x / n k ; a_{1} \bar{n}, q_{1}\right)
$$


we have

$$
\Delta_{l}(x ; a, q)=\sum_{\left(n, q_{1}\right)=1} F_{l}(n, k) \Delta_{l}\left(x / n k ; a_{1} \bar{n}, q_{1}\right) .
$$

We apply the bound from the hypothesis to the right-hand side here, assuming that $q_{1} \leq(x / n k)^{\kappa}$ for all $n$ in the range of summation, if such a restriction is required. Since $F_{l}(n, k)=0$ unless $n \mid k^{l-1}$, the strongest restriction is $q_{1} \leq\left(x / k^{l}\right)^{\kappa}$, and the worst bound on $\Delta_{l}\left(x / n k ; a_{1} \bar{n}, q_{1}\right)$ is $(x / k)^{\eta} q_{1}^{\theta}$. Since $F_{l}(n, k)$ and the number of terms in the sum in (6.2) are $\langle 1\rangle$, we get the stated result by recalling that $q_{1}=q / k$.

We finish by proving (6.1). If $(a, q)=1$ then the main term $M_{l}$ is simpler:

$$
M_{l}(x ; a, q)=\frac{1}{\phi\left(q_{1}\right)} \operatorname{Res}\left\{L^{l}\left(s, \chi_{0}\right) \frac{x^{s}}{s} ; s=1\right\} .
$$

Heath-Brown's argument in [H3], $\S 8$, uses this to show that the right-hand side of (6.1) is

$$
\frac{1}{\phi\left(q_{1}\right)} \operatorname{Res}\left\{\sum_{\substack{n \geq 1 \\(n, q)=k}} \frac{d_{l}(n)}{n^{s}} \frac{x^{s}}{s} ; s=1\right\} .
$$

Note that

so this becomes

$$
\sum_{\substack{n \geq 1 \\(n, q)=k}} \frac{d_{l}(n)}{n^{s}}=\sum_{\substack{a=1 \\(a, q)=k}}^{q} Z_{l}(s ; a, q)
$$

$$
\frac{1}{\phi\left(q_{1}\right)} \sum_{\substack{a=1 \\(a, q)=k}}^{q} M_{l}(x ; a, q) ;
$$

this is just $M_{l}(x ; a, q)$ since by (2.13) and Remark (II) following Lemma 4.2, $M_{l}(x ; a, q)$ depends only on $k$, and the number of $a \bmod q$ with $(a, q)=k$ is exactly $\phi(q / k)$.

If we apply this to Matsumoto's bound (5.2) then we get the bound

$$
\Delta_{l}(x ; a, q) \lll_{l}(x / k)^{(l-1) /(l+1)}
$$

provided $q \leq x^{2 /(l+1)} k^{(1-l) /(l+1)}$, which is satisfied for example when $q \leq$ $x^{1 / l}$.

Note from the proof that if we have a bound for $\Delta_{l}(x ; a, q)$ when $(a, q)=$ 1 which is a sum of terms of the form given in the proposition, then the bound when $(a, q)>1$ can be written as a sum of terms of the corresponding form. From this, if we apply Proposition 3 to the bound in Theorem 2, then we 
have

$$
\Delta_{l}(x ; a, q) \lll_{l}\left(\frac{x}{k}\right)^{(l-1) /(l+2)}+\left(\frac{x}{k}\right)^{1 / 2}\left(\frac{q}{k}\right)^{(l-4) / 4} ;
$$

the first term dominates the second when $q \leq x^{2 /(l+2)} k^{l /(l+2)}$.

\section{References}

[C] C. E. Chace, Writing integers as sums of products, doctoral dissertation, Columbia University, 1990.

[FI1] J. B. Friedlander and H. Iwaniec, The divisor problem for arithmetic progressions, Acta Arith. 45 (1985), 273-277.

[FI2] - - - Incomplete Kloosterman sums and a divisor problem, Ann. of Math. 121 (1985), 319-350.

[H1] D. R. Heath-Brown, Hybrid bounds for Dirichlet L-functions, Invent. Math. 47 (1978), 149-170.

[H2] - The fourth power moment of the Riemann zeta function, Proc. London Math. Soc. (3) 38 (1979), 385-422.

[H3] - , The divisor function $d_{3}(n)$ in arithmetic progressions, Acta Arith. 47 (1987), $29-56$.

[K] H. G. Kopetzky, Über die Größenordnung der Teilerfunktion in Restklassen, Monatsh. Math. 82 (1976), 287-295.

[L1] A. F. Lavrik, A functional equation for Dirichlet L-series and the problem of divisors in arithmetic progressions, Amer. Math. Soc. Transl. (2) 82 (1969), 47-65.

[L2] - On the principal term in the divisor problem and the power series of the Riemann zeta-function in a neighborhood of its pole, English transl. in Proc. Steklov Inst. Math. 1979, no. 3, 175-183.

[Ma] K. Matsumoto, A remark on Smith's result on a divisor problem in arithmetic progressions, Nagoya Math. J. 98 (1985), 37-42.

[Mn] H. L. Montgomery, Topics in Multiplicative Number Theory, Lecture Notes in Math. 227, Springer, 1971.

[Mo] Y. Motohashi, An asymptotic series for an additive divisor problem, Math. Z. 170 (1980), 43-63.

[N] W. G. Nowak, On the divisor problem in arithmetic progressions, Comment. Math. Univ. St. Pauli 33 (1984), 209-217.

[P] M. M. Petečuk, The sum of values of the divisor function in arithmetic progressions whose difference is a power of an odd prime, Math. USSR-Izv. 15 (1980), 145-160.

[S] R. A. Smith, The generalized divisor problem over arithmetic progressions, Math. Ann. 260 (1982), 255-268

[T] E. C. Titchmarsh, The Theory of the Riemann Zeta-Function, 2nd ed. revised by D. R. Heath-Brown, Clarendon Press, Oxford 1986.

DEPARTMENT OF MATHEMATICS

WILLIAMS COLLEGE

WILLIAMSTOWN, MASSACHUSETTS 01267, U.S.A. 
